\title{
Is There a Role for Molecular Tests in Diagnosis of Gastroenteritis in India?
}

\author{
${ }^{1}$ Priya Khanna, ${ }^{2} \mathrm{G}$ Gopal Rao
}

\begin{abstract}
How to cite this article: Khanna P, Rao GG. Is There a Role for Molecular Tests in Diagnosis of Gastroenteritis in India? J Gastrointest Infect 2018;8(1):3-4.
\end{abstract}

\section{Source of support: Nil}

\section{Conflict of interest: None}

Infectious diarrhea is a leading cause of death in children in the world and accounts for considerable morbidity in all ages.Diarrhea causes 1.3 million deaths worldwide and is the second leading cause of death in children under 5 years. ${ }^{1}$ UNICEF reported that diarrhea accounted for $9 \%$ of deaths in children under 5 years of age in India. ${ }^{2}$ Furthermore, it is estimated that in India, disabilityadjusted life-year (DALY) and healthy years of life lost due to disability (YLD) for 2016 to be 1,263,500,000 and 27,002,912.9 respectively. $^{3}$

Most cases of diarrhea in children and a majority of cases of diarrhea in adults are caused by infection. Infectious diarrhea or gastroenteritis is caused by a wide array of organisms. The common etiological causes of gastroenteritis include bacteria (Campylobacter, Salmonella spp, Shigella sp, Escherichia coli (ETEC, EIEC, EHEC and EPEC) Clostridium difficile, Vibrio, Aeromonas spp, Vibrio sp); viruses (rotavirus, norovirus, astrovirus, adenovirus (Type 40 and 41), sapovirus) and parasites (Cryptosporidium, Cyclospora cayatanesis, Isospora belli, Giardia lamblia, Entameoba histolytica, Blastocystitis hominis, Dientameoba fragilis). Although many of these infections are self- limiting, viral diarrhea in children under 5 years and acute bacterial diarrhea in all ages can be life threatening. A recent report found that rotavirus (20.4\%), followed by shigellosis (11.1\%), adenovirus (9.6\%) and cryptosporidium (7\%) were the commonest causes of death due to diarrheal diseases in children younger than 5 years in India. ${ }^{4}$ However, little is known about

\footnotetext{
${ }^{1,2}$ Clinical Microbiologists

1,2Department of Microbiology, Northwick Park Hospital, London North West University Hospital NHS Trust, United Kingdom

Corresponding Author: Priya Khanna, Department of Microbiology, Northwick Park Hospital, London North West University Hospital NHS Trust, Harrow, Middlesex HA1 3UJ, United Kingdom, e-mail: p.khanna@nhs.net
}

the prevalence and etiology of diarrhea in the general population.

Unfortunately, clinical diagnosis of gastroenteritis cannot be relied upon to make an etiological diagnosis of diarrhea. Laboratory support is necessary to identify the pathogen. Traditionally, diagnosis of gastrointestinal pathogens has included microscopy, culture, and rapid antigen tests for a few pathogens such as rotavirus and $C$. difficile toxin. These tests are labor intensive and can take up to 72-96 hours for the results to be available. However, in the past few years, several variations of polymerase chain reaction (PCR) called nucleic acid amplification tests (NAAT) have been developed in developed countries (USA and Europe) that can detect a majority of gastrointestinal pathogens. In this editorial, we discuss the role of molecular diagnostics for the detection of organisms causing gastroenteritis in India.

A variety of commercially available molecular tests for gastroenteritis have been licensed in the United States of America or Europe. These include xTAG® Gastrointestinal Pathogen Panel (GPP), Verigene Enteric Pathogens Nucleic acid test (EP), FilmArray Gastrointestinal (GI) Panel, Pro Gastro SSCS, Great Basin Stool Bacterial Pathogens Panel, BD MAX Extended Enteric Bacterial Panel, EntericBio® real time Panel (Seresep), RIDA® Gene (r-Biopharm), Seegene- Seeplex diarrhea ACE detection, Allplex ${ }^{\mathrm{TM}}$ Gastrointestinal Panel Assay, CLART EnteroBac and NanoCHIP Gastrointestinal panel.

Overall, these molecular tests are rapid, sensitive and specific; and enable the detection of many pathogens that may cause infective diarrhea. For example, 'FilmArray' GI panel targets 22 pathogens responsible for diarrheal disease, and this assay has been evaluated in several recent studies. The various molecular tests differ in the range of pathogens that they detect, platform used, and time taken to detect the pathogens. ${ }^{5-7}$ A large, multicenter study in Europe tested 709 stool samples by the FilmArray GI panel and identified that 384 (54.2\%) of these were positive for at least one pathogen. In contrast, conventional laboratory methods were positive in only $128(18.1 \%)$ samples. ${ }^{5}$ A key disadvantage of molecular tests is that they detect not only viable organisms but also detect bacteria and viruses that may be dead or not necessarily are the cause of infection. This presents the clinicians with the dilemma - how to interpret positive 
tests. Also, none of the currently available tests detect presence of antimicrobial resistance, which is important in management of some types of bacterial gastroenteritis and epidemiology.

In practical terms, the current molecular diagnostic methods require sophisticated instruments and trained manpower. Are these tests cost effective? Most studies were conducted in developed countries where the cost of the molecular tests is offset by high labour costs, hospitalization and nursing in single isolation rooms. These tests are expensive even in high income countries. In the United States, Binnicker ${ }^{5}$ recommended reserving molecular GI panels for immunocompromised patients, patients with prolonged diarrhea and critically ill patients to improve test utilisation and reduce inappropriate use of antibiotics. The National Institute of Clinical Excellence (NICE) in the UK recently reviewed Multiplex PCR tests (xTAG Gastrointestinal Pathogen Panel, Film Array GI Panel and Fecal Pathogens B assay). It concluded that there is insufficient evidence for routine adoption of these tests in the National Health Service, based on costeffectiveness modelling. ${ }^{8}$

In general terms, healthcare challenges faced in LMIC differ considerably from those developed countries. Health expenditure as a percentage of GDP is 3.9\% in India as compared to $16.8 \%$ for USA, $9.9 \%$ for United Kingdom and $11.2 \%$ for Germany. ${ }^{9}$ There is also huge variation in the laboratory facilities available in India, ranging from small ill-equipped laboratories to those with cutting edge diagnostic facilities. In LMIC with limited healthcare budgets, it is generally not possible to perform all necessary microbiology tests leading to misdiagnosis and inappropriate treatment.

So, is there a place for molecular tests for detection of gastrointestinal pathogens in LMIC such as India? We believe that in India, molecular tests can have an important role in the investigation of intractable cases of gastroenteritis and diarrhea and in epidemiological studies to inform public health interventions. Molecular tests can help to differentiate between viral, bacterial and parasitic causes of diarrhea and in turn, may reduce indiscriminate use of antimicrobials in treatment of diarrhea. However, it will be important to have guidelines for the appropriate use of these tests to prevent overuse. Needless to say, robust quality assurance processes will also be required to prevent cross contamination and erroneous results.

In conclusion, while molecular tests can undoubtedly improve the etiological diagnosis of gastroenteritis, it is not yet clear if these expensive tests will alter the outcome of gastroenteritis. Management of gastroenteritis is mainly symptomatic even where an etiological diagnosis is made as the diarrhea is usually self-limiting. In LMIC like India, these tests are likely to be used in research or in the private health sector. The most effective use will be in epidemiological studies and in investigation and control of hospital and community outbreaks of gastroenteritis and food poisoning.

\section{REFERENCES}

1. WHO Fact sheet- Diarrhoeal disease. http://www.who.int/ news-room/fact-sheets/detail/diarrhoeal-disease (accessed 5-10-18).

2. UNICEF: Commission on Child Survival. A Promise Renewed. https:/ / www.unicef.org/publications / files / APR_2015_9_ Sep_15.pdf (accessed 05-05 2018).

3. Burden of Disease in India. http://www.who.int/macrohealth/action/NCMH_Burden of disease_(29 Sep 2005).pdf (accessed 05--05 2018).

4. GBD Disease Collaborators. Estimates of global, regional, and national morbidity, mortality, and aetiologies of diarrhoeal diseases: a systematic analysis for the Global Burden of Disease Study 2015. Lancet Infect Dis. 2017 Nov;17(11):1133-1161.

5. Binnicker MJ. Multiplex Molecular Panels for Diagnosis of Gastrointestinal Infection: Performance, Result Interpretation, and Cost-Effectiveness. J Clin Microbiol. 2015 Dec;53(12):3723-3728.

6. Zhang H, Morrison S, Tang Y-W. Multiplex polymerase chain reaction tests for detection of pathogens associated with gastroenteritis.Clin Lab Med. 2015 Jun;35(2):461-867.

7. Reddington K, Tuite N, Minogue E, Barry T. A current overview of commercially available nucleic acid diagnostics approaches to detect and identify human gastroenteritis pathogens. Biomol Detect Quantif. 2014 Aug 14;1(1):3-7.

8. Integrated multiplex PCR tests for identifying gastrointestinal pathogens in people in suspected gastroenteritis (xTAG Gastrointestinal Pathogen PAnel, FilmArray GI Panel and Faecal Pathogens B Assay. Diagnostics guidance (DG 26) Published date January 2017 https://www.nice.org.uk/ guidance/dg26 (Accessed 15-11-18).

9. Global Health Observatory (GHO) Data. Current health expenditure as a percentage of gross domestic product (GDP)https:/ /www.who.int/gho/health_financing/health_ expenditure/en/(Accessed 16-12-2018). 